\title{
pH Response of Silicon Nanowire Sensors: Impact of Nanowire Width and Gate Oxide
}

\author{
Kristine Bedner*, Vitaliy Anatolijovic Guzenko, Alexey Tarasov ${ }^{1}$, Mathias Wipf ${ }^{1}$, \\ Ralph Lukas Stoop ${ }^{1}$, David Just ${ }^{1}$, Sara Rigante ${ }^{2}$, Wangyang $\mathrm{Fu}^{1}$, \\ Renato Amaral Minamisawa, Christian David, Michel Calame ${ }^{1}$, \\ Jens Gobrecht and Christian Schönenberger ${ }^{1}$
}

Laboratory for Micro- and Nanotechnology, Paul Scherrer Institute, PSI Villigen, Switzerland ${ }^{1}$ Department of Physics, University of Basel, Basel, Switzerland

École Polytechnique Fédéral de Lausanne, Lausanne, Switzerland

(Received January 9, 2013; accepted May 9, 2013)

Key words: $\quad \mathrm{pH}$ response, nanowire sensor, gate oxide, ion-sensitive field-effect transistor

We present a systematic study of the performance of silicon nanowires (SiNWs) with different widths when they are used as ion-sensitive field-effect transistors (ISFETs) in $\mathrm{pH}$-sensing experiments. The SiNW widths ranged from $100 \mathrm{~nm}$ to $1 \mu \mathrm{m}$. The SiNWISFETs were successfully fabricated from silicon-on-insulator (SOI) wafers with $\mathrm{Al}_{2} \mathrm{O}_{3}$ or $\mathrm{HfO}_{2}$ as gate dielectric. All the SiNWs showed a pH Response close to the Nernstian limit of $59.5 \mathrm{mV} / \mathrm{pH}$ at $300 \mathrm{~K}$, independent of their width, or the investigated gate dielectric or operating mode. Even nanowires (NWs) in the $100 \mathrm{~nm}$ range operated reliably without degradation of their functionality. This result is of importance for a broad research field using SiNW sensors as a candidate for future applications.

\section{Introduction}

In recent years, the ion-sensitive field-effect transistor (ISFET) ${ }^{(1,2)}$ has been scaled down to a silicon nanowire ISFET (SiNW-ISFET) with sub-100 dimensions. ${ }^{(3-6)}$ The reduced size offers unique possibilities, such as the dense integration of multiplexed NWs, ${ }^{(7,8)}$ which sense a large number of different target molecules simultaneously using differently functionalized NWs.(9) The key feature for biological applications is that nanostructures are on the scale of biological specimens. Recently, Tian et al.(10) have demonstrated a manipulation of the living cell with nano-FETs without damaging the cell.

${ }^{*}$ Corresponding author: e-mail: Kristine.Bedner@psi.ch 
The sensing mechanism of SiNW-ISFETs is based on the accumulation of charged molecules on the NW surface, which leads to a surface potential shift. The transistor then responds to changes in the surface potential with a threshold voltage shift $\Delta V_{\text {th }}$. In a pH-sensing experiment, the accumulated surface charge originates from $\mathrm{OH}$ groups located on the surface of the gate dielectric, which are positively (protonated) or negatively (deprotonated) charged, according to the $\mathrm{pH}$ of the buffer solution. This surface charge provokes the $\mathrm{pH}$ response of the SiNW-ISFET in the form of a threshold voltage shift. Consequently, the $\mathrm{pH}$ response is given in $\mathrm{mV} / \mathrm{pH}$. Several publications addressed the question on whether the NW width $^{(4,11-13)}$ or thickness ${ }^{(14,15)}$ has an effect on the $\mathrm{pH}$ response; however, the results were inconsistent. An effect of the NW width on the response in $\mathrm{pH}$-sensing experiments has been shown in refs. 12 and 13. Elfström et $a l .{ }^{(12)}$ observed an increasing threshold voltage shift with decreasing $\mathrm{NW}$ width. No $\mathrm{pH}$ response was found for NW widths $>150 \mathrm{~nm}$. Vu et al. ${ }^{(4)}$ reported preliminary results for single 80- and 400-nm-wide wires that exhibit the same threshold voltage shift of $41 \mathrm{mV} / \mathrm{pH}$. Moreover, the ideal working point showing the highest $\mathrm{pH}$ response is also a controversial discussion in the literature. Gao et al.(16) demonstrated an enhancement of the $\mathrm{pH}$ response in the subthreshold regime compared with that in the linear regime, whereas preliminary results described in ref. 4 suggest the same $\mathrm{pH}$ response in both regimes. To clarify these disagreements, we explore in a systematic manner the effect of the NW width of SiNW-ISFETs on the $\mathrm{pH}$ response with widths ranging from 1 $\mu \mathrm{m}$ down to $100 \mathrm{~nm}$ in both operating modes, the subthreshold and linear modes. In addition, we study the sensing properties of the SiNW-ISFET coated with $\mathrm{Al}_{2} \mathrm{O}_{3}$ and $\mathrm{HfO}_{2}$ grown by atomic layer deposition (ALD). Compared with $\mathrm{SiO}_{2},{ }^{(4,10,11,16)}$ these oxides provide a leakage-free ${ }^{(17)}$ operation with an ideal Nernstian $\mathrm{pH}$ response that is independent of the salt concentration. ${ }^{(5,6,18)}$ For the application of wide $\mathrm{pH}$ ranges, $\mathrm{HfO}_{2}$ is a predestined candidate as a sensing surface due to its chemical inertness in most acidic or basic solutions.

\section{Materials and Methods}

The SiNW-ISFETs were fabricated from silicon-on-insulator (SOI) wafers following the top-down approach. ${ }^{(11)}$ The SOI wafer (Soitec, France) has a buried oxide layer of $145 \mathrm{~nm}$ and an 85-nm-thick low-doped p-Si(100) device layer with a resistivity of 8.5 to $11.5 \Omega \cdot \mathrm{cm}$. First, the Si device layer was thermally oxidized with a $15-\mathrm{nm}$-thick $\mathrm{SiO}_{2}$ layer. Then, the pattern of the SiNW-ISFET was defined by electron beam lithography (EBL) in a negative resist (AZ nLOF 2000) and transferred to the $\mathrm{SiO}_{2}$ layer by reactive ion etching. The patterned $\mathrm{SiO}_{2}$ was used as an etching mask for the wet chemical etching of the Si device layer in tetra-methyl ammonium hydroxide (TMAH) with 10 vol.\% isopropanol (IPA) at $45{ }^{\circ} \mathrm{C}$. The wet chemical etching of the Si(100) layer is highly anisotropic with etch rates depending on the crystallographic orientation of the silicon. ${ }^{(19)}$ The Si(111) planes are etched approximately 100 times slower ${ }^{(11)}$ than the other planes, which results in a trapezoidal shape of the NW cross section. A key feature of our sensors is that a single SiNW-ISFET chip contains $48 \mathrm{NWs}$ with eight top widths $W_{\text {top }}$ [Fig. 1(a)] ranging from $1 \mu \mathrm{m}$ down to $100 \mathrm{~nm}$. The $48 \mathrm{NWs}$ are arranged in four arrays with twelve NWs sharing the common drain contact. The length of the NWs 

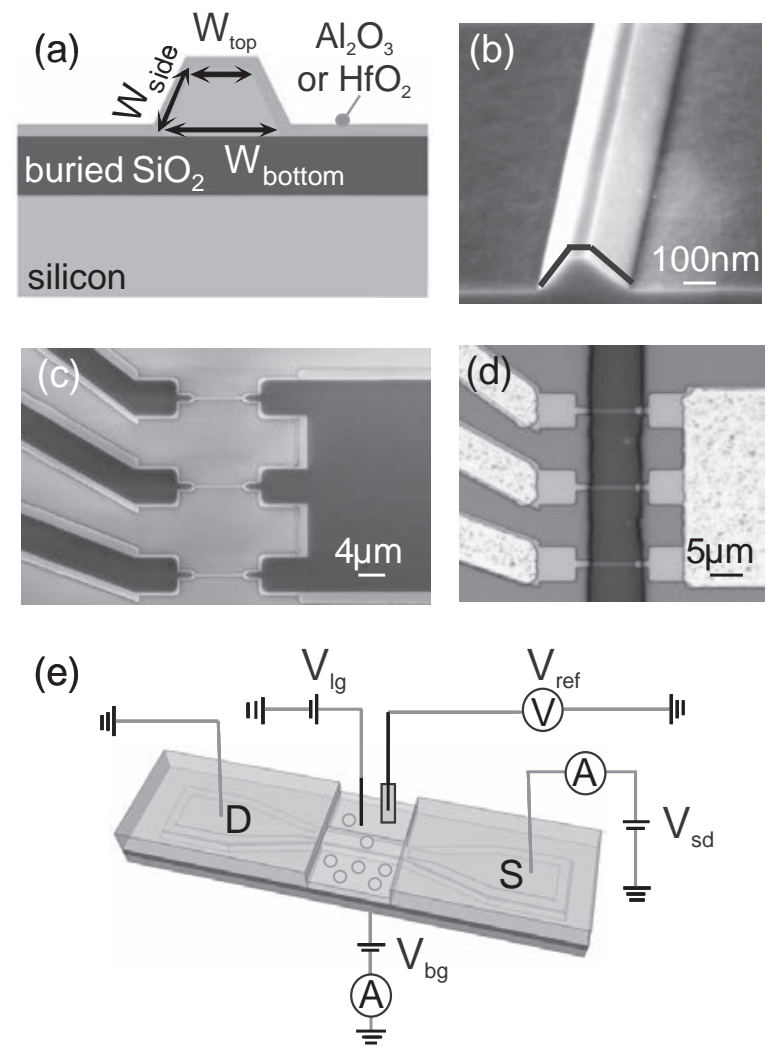

Fig. 1. (a) Schematic cross section of a wet-etched silicon NW fabricated from a (100) SOI wafer. It shows the top and bottom widths of the NW, $W_{\text {top }}$ and $W_{\text {bottom, respectively. The sidewalls }}$ $W_{\text {side }}$ are formed by the $\mathrm{Si}(111)$ planes. As top oxide, $\mathrm{Al}_{2} \mathrm{O}_{3}$ or $\mathrm{HfO}_{2}$ was used, grown by ALD. The thicknesses of the ALD oxides were $20 \mathrm{~nm}$ for $\mathrm{Al}_{2} \mathrm{O}_{3}$ and 10 or $20 \mathrm{~nm}$ for $\mathrm{HfO}_{2}$. (b) Electron beam micrograph of a NW cross section. The solid blue line represents the effective wire width, $W_{\text {eff }}=W_{\text {top }}+2 W_{\text {side }}$. (c) Electron beam micrograph of a NW array showing three NWs $\left(W_{\text {top }}=\right.$ $100 \mathrm{~nm}$ ) connected to the implanted (dark area) source contacts on the left and the common drain contact on the right. (d) Micrograph of a NW array with three NWs ( $W_{\text {top }}=100 \mathrm{~nm}$ ) connected to the implanted contacts, which are covered by metal. SU-8 resist is used to form the vertical liquid channels on top of the NW array. (e) Schematics of the liquid measurement setup. A source-drain current $I_{\text {sd }}$ flows through the NW as the source-drain voltage $V_{\text {sd }}$ is applied. The external voltages $V_{\mathrm{bg}}$ and $V_{\mathrm{lg}}$ can be applied to the back gate and liquid gate, respectively. The reference electrode determines the potential $V_{\text {ref }}$ in the liquid.

is $6 \mu \mathrm{m}$ and their height is about $85 \mathrm{~nm}$. The bottom and sidewall widths are denoted as $W_{\text {bottom }}$ and $W_{\text {side }}$, respectively [Fig. 1(a)]. The top perimeter of the NW comprising the sum of $W_{\text {top }}$ and both sidewalls is represented by the effective wire width, $W_{\text {eff }}$ [Fig. 1(b)]. After the silicon etching, the pattern of the source and drain contacts was defined 
in the PMMA resist by EBL. The resist acted as an implantation mask for the following implantation with a $\mathrm{BF}_{2}{ }^{+}$dopant dose of $2.3 \times 10^{15} \mathrm{~cm}^{-2}$ at $33 \mathrm{keV}$. The implanted area approached the NW to a distance of $\sim 200 \mathrm{~nm}$. The NW retained its original low p-type doping concentration. After implantation, the dopants were activated in a thermal annealing step at $950{ }^{\circ} \mathrm{C}$ for 6 min in forming gas. The NW array with three NWs connected to the implanted source and common drain contact is shown in Fig. 1(c). The passivation of the SiNW-ISFET, which is necessary for measurements in liquids, was carried out by atomic layer deposition (ALD) of $\mathrm{Al}_{2} \mathrm{O}_{3}$ and $\mathrm{HfO}_{2}$ (Savannah S100 Cambridge NanoTech). The ALD oxides were deposited directly on the NW surface. Immediately prior to the ALD oxide growth, a Radio Corporation of America (RCA) cleaning was done. During the RCA cleaning, the 15-nm-thick thermal $\mathrm{SiO}_{2}$ was etched in buffered hydrofluoric acid (BHF). After the growth of the ALD oxides, the pattern of the source/drain contact windows was defined by optical lithography in the resist AZ nLOF 2000. The contact windows were then opened in BHF. The etch rate of $\mathrm{Al}_{2} \mathrm{O}_{3}$ is comparable to that of thermal $\mathrm{SiO}_{2}$, whereas $\mathrm{HfO}_{2}$ etches 14 times slower. Immediately after the etching, the opened contacts were metalized with $\mathrm{Al}-\mathrm{Si}(1 \%)$ in a lift-off process. The formation of the ohmic contacts was completed in a thermal annealing process in forming gas at $450{ }^{\circ} \mathrm{C}$. In the same step, the ALD oxides were also postannealed. It is known that the postannealing of the ALD oxides in forming gas has the effect of reducing the density of interface states $D_{\mathrm{it}}$ as they are passivated by hydrogen. ${ }^{(20)}$ Also, the dielectric constants of the oxides are affected as the oxide structure changes from amorphous to (poly)-crystalline, depending on the annealing temperature. However, the ALD oxides have a large excess of $\mathrm{O}_{2}$ as they are grown in an oxygen-rich environment. Therefore, there is the risk that during the postannealing, the excess $\mathrm{O}_{2}$ diffuses to the $\mathrm{Si}$ where it leads to the growth of the interfacial $\mathrm{SiO}_{2}$ layer ${ }^{(20,21)}$ that already formed between the Si substrate and the ALD oxide during the ALD deposition. ${ }^{(20)}$ The smaller dielectric constant of 3.9 for $\mathrm{SiO}_{2}$ will reduce the total dielectric constant of the ALD layer. To integrate the fluidic system, microchannels were fabricated in the SU-8 resist by optical lithography. Figure 1(d) shows a micrograph of the SiNW-ISFET after this process step. One microchannel aligned perpendicular to the NWs can be seen. After the wire bonding, the contact pads including the wire bonds were sealed with epoxy. In the experiment, the microchannel was filled with an electrolyte, and the electrostatic potential of this electrolyte was mediated through the liquid to the NWs. This is known as liquid gating. The $\mathrm{pH}$-sensing measurements were carried out in standard $\mathrm{pH}$ buffer solutions (Titrisol, Merck) with different $\mathrm{pH}$ values. In Fig. 1(e), a schematic of the measurement setup is shown. ${ }^{(17,22)}$ The device was operated at a source-drain voltage $V_{\text {sd }}$ of $0.1 \mathrm{~V}$ and the conductance $G$ through the device was measured. The conductance was modulated by a top liquid gate voltage $V_{\mathrm{lg}}$ that was applied to a platinum wire immersed in the buffer solution. A calomel reference electrode was used to measure the effective potential $V_{\text {ref }}$ in the buffer solution against ground. Additionally, a back gate voltage $V_{\mathrm{bg}}$ can be applied to the back side of the sample. The measurements were performed with buffer solutions with $\mathrm{pH}$ values ranging from 3 to 9 and from 3 to 10 , for $\mathrm{Al}_{2} \mathrm{O}_{3}$ and $\mathrm{HfO}_{2}$, respectively. For $\mathrm{Al}_{2} \mathrm{O}_{3}$ the highest $\mathrm{pH}$ was 9 as the $\mathrm{Al}_{2} \mathrm{O}_{3}$ is etched at higher $\mathrm{pH}$ values. The basic buffers did not attack the $\mathrm{HfO}_{2}$ layer due to its chemical inertness to 
acidic or basic solutions, apart from hydrofluoric (HF) acid-based solutions. We started with the buffer at $\mathrm{pH} 3$ and exchanged sequentially the buffers with steps of $\Delta p H=1$ until the buffer with the highest $\mathrm{pH}$ was reached. We then returned stepwise to $\mathrm{pH} 3$. All the 48 NWs of one SiNW-ISFET chip were measured automatically at different $\mathrm{pH}$ values with a LabView-controlled measurement setup including a switching box (Keithley 3706).

\section{Results and Discussion}

Figure 2(a) shows the conductance $G$ on a logarithmic scale as a function of reference voltage $V_{\text {ref }}$ for NWs with $W_{\text {top }}$ of $100 \mathrm{~nm}$ and $1 \mu \mathrm{m}$, measured at pHs 4, 6, and 8. The hysteresis in the transfer curves for the forward and backward voltage sweep of $V_{\text {ref }}$ is small and maximum $5 \mathrm{mV}$. The transfer curves shift to more positive $V_{\text {ref }}$ values if the $\mathrm{pH}$ is increased, ${ }^{(8)}$ which is in agreement with the site-binding model.(2) Here, to determine the $\mathrm{pH}$ response, we do not extract the threshold voltage $V_{\text {th }}$ according to

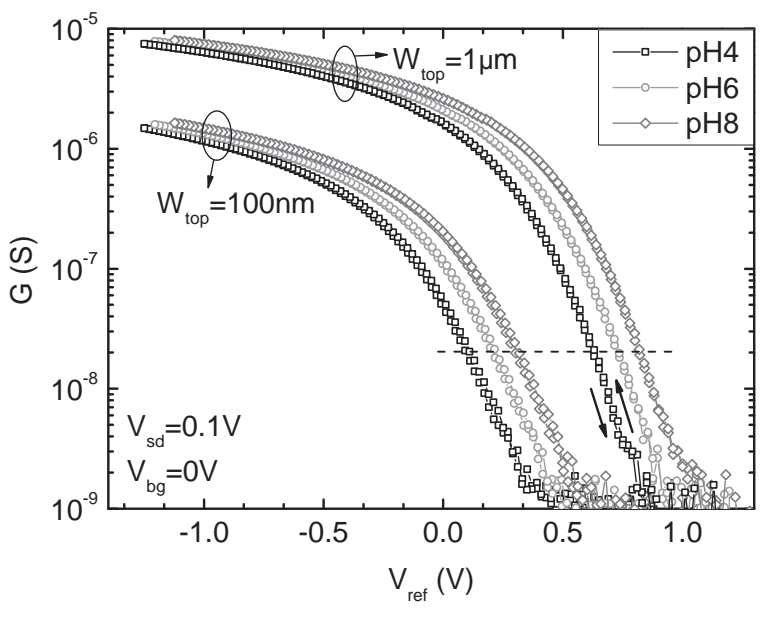

(a)

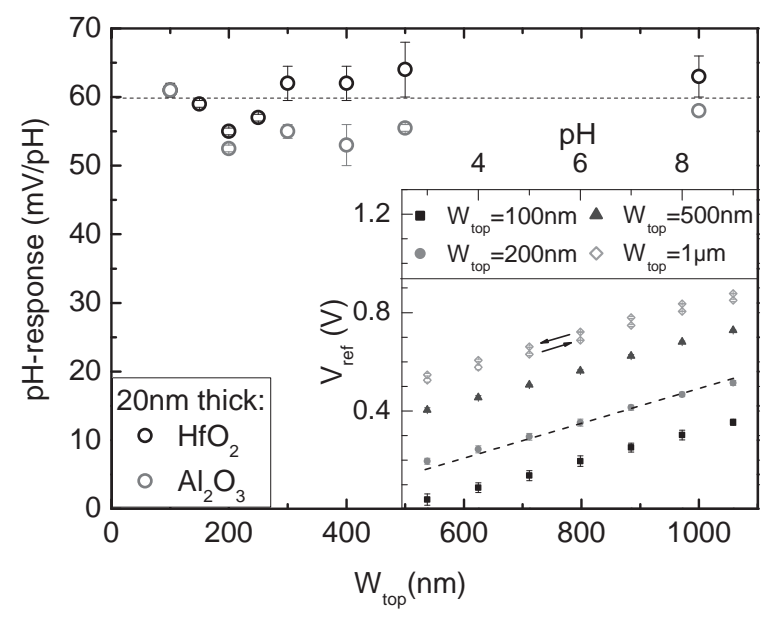

(b)

Fig. 2. (a) Logarithmic conductance $G$ vs reference voltage $V_{\text {ref }}$ for two NWs $\left(W_{\text {top }}=100 \mathrm{~nm}\right.$ and $1 \mu \mathrm{m}$ ) measured at $\mathrm{pHs} 4,6$, and 8 . Owing to the $\mathrm{pH}$ response, the transfer curves shift to the right with increasing $\mathrm{pH}$. The transfer curves have a small hysteresis of maximum $5 \mathrm{mV}$ in the forward and backward sweeps of $V_{\text {ref. }}$ (b) The $\mathrm{pH}$ response of NWs with $W_{\text {top }}$ ranging from $100 \mathrm{~nm}$ to 1 $\mu \mathrm{m}$ is close to the Nernst limit of $59.5 \mathrm{mV} / \mathrm{pH}$ at $300 \mathrm{~K}$ (black, dashed line). Buffer solutions with $\mathrm{pH}$ values from 3 to 9 for $\mathrm{Al}_{2} \mathrm{O}_{3}$ were used. An effect of the $\mathrm{NW}$ width or gate oxide on the $\mathrm{pH}$ response is not observed. Inset: The liquid potential $V_{\text {ref }}$ extracted at $20 \mathrm{nS}$ [Fig. 2(a) dashed line] is plotted as a function of $\mathrm{pH}$. A linear dependence is found over the whole $\mathrm{pH}$ range. The $\mathrm{pH}$ response is defined by the slope of the best fit line. The NW with $W_{\text {top }}=1 \mu \mathrm{m}$ shows the $\mathrm{pH}$ response for $\mathrm{pH}$ values measured from 3 to 9 and back to 3 with a negligible hysteresis. 
one of the classical methods, ${ }^{(23)}$ as we are not interested in the absolute $V_{\text {th }}$ values. It is sufficient to know the relative shift of the transfer curves. Therefore, we defined $V_{\text {th }}$ as the reference potential $V_{\text {ref }}$ at any source-drain conductance value in the subthreshold regime. In our experiments, this value was $20 \mathrm{nS}$ as indicated by the dashed line in the semilogarithmic plot of Fig. 2(a). Consequently, the threshold voltage shift $\Delta V_{\text {th }}$ was replaced by $\Delta V_{\text {ref }}$. A set of $V_{\text {ref }}$ values at $20 \mathrm{nS}$ for different $\mathrm{pH}$ values are presented for four NW widths in the inset of Fig. 2(b). A linear dependence is found over the whole $\mathrm{pH}$ range. Moreover, a small negligible hysteresis in the forward and backward sweeps of the $\mathrm{pH}$ values is observed. (The drift rate of $V_{\text {ref }}$ is a few $\mathrm{mV} / \mathrm{h}$ for samples covered with 20-nm-thick $\mathrm{Al}_{2} \mathrm{O}_{3}$.) The slope of a linear fit to the data defines the $\mathrm{pH}$ response of the $\mathrm{NW}$ in $\mathrm{mV} / \mathrm{pH}$. These slopes are shown in Fig. 2(b) for NWs with $W_{\text {top }}$ ranging from $100 \mathrm{~nm}$ to $1 \mu \mathrm{m}$. All the NWs show an ideal Nernstian behavior of $60 \mathrm{mV} / \mathrm{pH}$ at $300 \mathrm{~K}$, independent of the NW width or gate oxide type and without degradation of the $\mathrm{pH}$ response over time. To the best of our knowledge, this is the first systematic study showing a clear absence of any size dependence on $\mathrm{pH}$-sensing properties of NWs. The observed ideal $\mathrm{pH}$ response confirms recent results showing that ALD-grown $\mathrm{Al}_{2} \mathrm{O}_{3}{ }^{(18)}$ and $\mathrm{HfO}_{2}{ }^{(6)}$ are excellent pH-sensing interfaces, owing to the high density of active surface groups that buffer the $\mathrm{pH}$ changes in the bulk solution. Within the site-binding model, ${ }^{(2)}$ this oxide property is called the surface buffer capacity. As long as the oxide/ electrolyte interface of the surface of the NW provides a large surface buffer capacity for protons, no size dependence is expected. Our observation is therefore in strong contrast to the work described in refs. 12 and 13.

In an application, it is convenient to measure a large conductance. For fast readout, it is therefore beneficial to operate the ISFETs in the linear regime and not in the subthreshold regime. In the linear regime, the measured signal is the conductance change $\Delta G(\mathrm{pH})$ at constant $V_{\text {ref. }}$. It can be converted to a voltage shift $\Delta V_{\text {ref }}$ using the transconductance $g_{\mathrm{m}}=\mathrm{d} G / \mathrm{d} V_{\text {gate }}$ :

$$
\Delta G(\mathrm{pH})=g_{\mathrm{m}} \Delta V_{\text {ref }}
$$

To investigate the influence of the NW width on the response of $\Delta G$ to $\mathrm{pH}$ changes in the linear regime, one has to carefully analyze the transconductance $g_{\mathrm{m}}$. A comparison between different NW widths requires a normalization of the conductance because it changes with the wire size and shape. ${ }^{(24,25)}$ Different methods could be assumed, such as normalization with the cross-sectional area of the wire, the top width $W_{\text {top }}$, the top perimeter $W_{\text {eff }}=W_{\text {top }}+2 W_{\text {side }}$, the bottom width $W_{\text {bottom }}$, or even the full perimeter of the wire $W_{\text {eff }}+W_{\text {bottom }}$. It is not clear a priori which method is the best, because we do not know the exact current distribution along the NW cross section. If the current flows homogeneously over the full cross section, one would have to scale with the area. There are many reasons, however, why this might not be the case. For example, we can gate the NW asymmetrically and thereby accumulate more carriers at the top surface as compared with the bottom surface, or vice versa. It is also known that surfaces with different crystallographic orientations have different band alignments; ${ }^{(21)}$ hence, the accumulation of carriers may start differently at different crystal faces. Since a detailed systematic 
study of these variabilities is beyond the scope of this work, we assume that the current scales with the top perimeter, i.e., $W_{\text {eff }}=W_{\text {top }}+2 W_{\text {side }}$. This assumption is motivated by the gating scheme via the liquid gate (top gate), which is used in the experiments in this work. It is further justified, because the electrolyte is in contact simultaneously with both the top and side faces. We thereby neglect the possible dependence of the current on the crystal orientation.

In Fig. 3(a), the transfer curves normalized by $W_{\text {eff }}$ are shown for two NWs with $W_{\text {top }}$ of $100 \mathrm{~nm}$ and $1 \mu \mathrm{m}$ at different $\mathrm{pH}$ values. The linear regimes of both normalized transfer curves are very similar, which indicates an identical scaled transconductance for different wire widths. Moreover, the scaled transconductance values for a set of NWs with different $W_{\text {top }}$ and $\mathrm{HfO}_{2}$ or $\mathrm{Al}_{2} \mathrm{O}_{3}$ as gate oxide are shown in Fig. 2(b). The error bars result from an average over two NWs with an equivalent $W_{\text {top }}$. All the NWs with different widths show a similar scaled transconductance. On the basis of this result, we can conclude that in the linear regime, the normalized conductance change $\Delta G$, and therefore, the response to $\mathrm{pH}$ changes, is also independent of the $\mathrm{NW}$ width and the operating regime. Our result is in contrast with the results shown by Gao et al. ${ }^{(16)}$ Therefore, our results prove that one can fabricate NW sensors with ideal Nernstian behaviour with NWs down to $100 \mathrm{~nm}$ width, and an operation in the linear and subthreshold regimes is favourable.

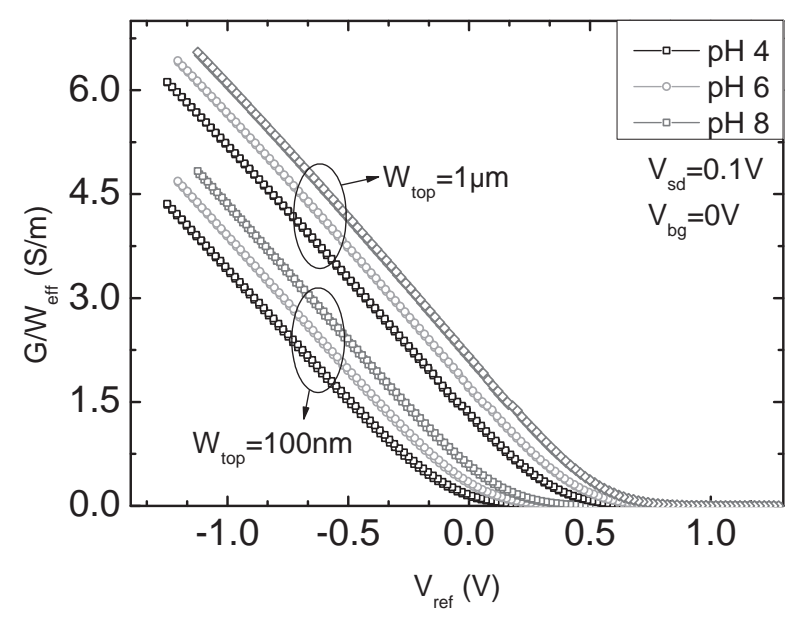

(a)

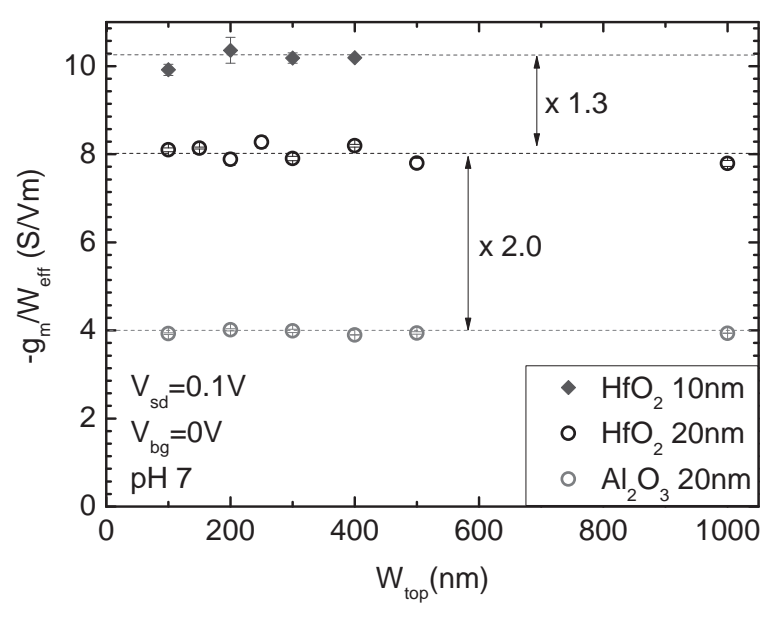

(b)

Fig. 3. (a) Conductance $G$, normalized with the effective wire width $W_{\text {eff }}$ vs the reference voltage $V_{\text {ref }}$ for two NWs ( $W_{\text {top }}=100 \mathrm{~nm}$ and $1 \mu \mathrm{m}$ ) measured at pHs 4, 6, and 8. (b) Scaled transconductance $g_{\mathrm{m}} / W_{\text {eff }}$, extracted from the normalized conductance in Fig. 3(a) for different NW widths and gate oxides. It is constant for NW with $W_{\text {top }}$ between $100 \mathrm{~nm}$ and $1 \mu \mathrm{m}$. The dashed line is the calculated average value. The transconductance changes with the oxide type and thickness. 
Although the scaled transconductance values are constant for wires of different widths, we have to understand the different $g_{\mathrm{m}} / W_{\text {eff }}$ values for different top gate oxides. In the linear regime, $g_{\mathrm{m}}$ is determined by the mobility $\mu$ of the charge carriers and the liquid gate capacitance per unit area, $C_{\mathrm{lg}}$ :

$$
g_{\mathrm{m}}=\mu C_{\mathrm{lg}} W_{\text {eff }} / L
$$

Here, $L$ denotes the length of the NWs, which was constant for all the wires. We assume that for one sample, the mobility is constant for all the wires of different widths as it is indicated by the constant value of the scaled transconductance. Sample-to-sample variations in the mobility may still occur in the fabrication process. Therefore, the scaling of $g_{\mathrm{m}}$ with $W_{\text {eff }}$ will then equally be reflected in the capacitance. As a consequence, the value of the scaled transconductance $g_{\mathrm{m}} / W_{\text {eff }}$ should be determined using $C_{\mathrm{lg}}$. The liquid gate voltage is applied to the NW through the liquid via the top oxide (TOX). It, therefore, involves the double-layer $C_{\mathrm{dl}}$ in the electrolyte and the top-oxide capacitance $C_{\mathrm{TOx}}$ connected in series. Owing to the high ion concentration in the electrolyte of $100 \mathrm{mM}$, we have $C_{\mathrm{dl}}>>C_{\mathrm{TOX}}$. In the series connection, this leads to $C_{\mathrm{lg}} \approx C_{\mathrm{TOX}}$ and

$$
g_{\mathrm{m}} / W_{\text {eff }}=\mu C_{\mathrm{TOX}} / L
$$

Looking at Fig. 3(b), one would naively expect a factor of two difference in the measured $g_{\mathrm{m}} / W_{\text {eff }}$ values between the $\mathrm{HfO}_{2}$ samples with 10 and $20 \mathrm{~nm}$ thicknesses, instead of 1.3. To resolve this discrepancy, we determined the dielectric constants $\varepsilon_{\mathrm{r}}$ by capacitance-voltage $(C-V)$ spectroscopy. We obtained $\varepsilon_{\mathrm{r}}=12$ and 17 for $\mathrm{HfO}_{2}$ layers with 10 and $20 \mathrm{~nm}$ thicknesses, respectively. The 20-nm-thick $\mathrm{Al}_{2} \mathrm{O}_{3}$ layer had a dielectric constant $\varepsilon_{\mathrm{r}}=7.2$. Our results are comparable to the reported values of $\varepsilon_{\mathrm{r}}=10$ $-18^{(26)}$ and $\varepsilon_{\mathrm{r}}=4-10^{(21)}$ for $\mathrm{HfO}_{2}$ and $\mathrm{Al}_{2} \mathrm{O}_{3}$, respectively. From our $C-V$ measurements, we see that the dielectric constants are thickness dependent as suggested by the $g_{\mathrm{m}}$ values of the $\mathrm{HfO}_{2}$ data in Fig. 3(b). The reason for this might be the presence of an interfacial $\mathrm{SiO}_{2}$ layer of $2 \mathrm{~nm}$ maximum thickness, which grows during the ALD process ${ }^{(20)}$ between the Si substrate and the ALD oxides. It reduces the dielectric constant of the total layer, according to the series capacitance of the $\mathrm{SiO}_{2}$ and the ALD oxide layer. Knowing the dielectric constants of $\mathrm{HfO}_{2}$ layers, we estimated a $C_{\mathrm{TOx}}$ capacitance ratio of 1.4 between the 10 - and 20 -nm-thick $\mathrm{HfO}_{2}$ samples. Indeed, this result shows that we cannot expect a factor of two difference in the measured $g_{\mathrm{m}} / W_{\text {eff }}$ values in Fig. 3(b). The slight discrepancy between the $C_{\text {Tox }}$ capacitance ratio, which is 1.4 , and the ratio of 1.3 for $g_{\mathrm{m}} / W_{\text {eff }}$ can be attributed to slight differences in the mobility. On the basis of our data, we estimated mobilities of 58 and $62 \mathrm{~cm}^{2} /$ Vs for the 10- and 20-nm-thick $\mathrm{HfO}_{2}$ samples, respectively. For the $\mathrm{Al}_{2} \mathrm{O}_{3}$ sample, we obtained an estimated hole mobility of $75 \mathrm{~cm}^{2} /$ Vs. The mobility values are in good agreement with the reported hole mobilities for SOI wafers in the accumulation mode (50-139 $\left.\mathrm{cm}^{2} / \mathrm{Vs}\right)$, ${ }^{(1)}$ with similar doping concentrations. The slightly reduced mobility observed in both samples covered with $\mathrm{HfO}_{2}$ is in agreement with previous results in the literature, which report a mobility degradation in MOSFETs fabricated with high- $\kappa$ materials. ${ }^{(27)}$ 


\section{Conclusions}

In conclusion, we have systematically studied the effect of the NW width $W_{\text {top }}$ ranging from $100 \mathrm{~nm}$ to $1 \mu \mathrm{m}$ on the $\mathrm{pH}$ response of SiNW-ISFET. Here, the $\mathrm{pH}$ response is determined from the voltage shift $\Delta V_{\text {ref }}$ of the transfer curve in $\mathrm{mV} / \mathrm{pH}$. SiNW-ISFETs with $\mathrm{Al}_{2} \mathrm{O}_{3}$ or $\mathrm{HfO}_{2}$ exhibit an ideal linear Nernstian response of $60 \mathrm{mV} / \mathrm{pH}$ at $300 \mathrm{~K}$ in the subthreshold and linear regimes, without degradation of the Nernstian $\mathrm{pH}$ response over time. The drift rate of $V_{\text {ref }}$ is a few $\mathrm{mV} / \mathrm{h}$. For future experiments, we suggest to correct and thereby minimize the influence of drift on the sensor response by differential measurements, where a passivated NW is used as the reference NW.(28) No effect of the NW width on the $\mathrm{pH}$ response was observed. The results of our systematic study are in strong contrast to those described in previous publications as no effect of the wire width or the operating regime on the $\mathrm{pH}$ response is observed. Not only the sensing properties but also the electrical properties of the NWs are exceptionally good and reproducible, e.g., the $48 \mathrm{NWs}$ integrated on one sample have a variation in $V_{\text {th }}$ of $\sim 50 \mathrm{mV}$ from NW to NW of the same width. Also, the negligible hysteresis in the transfer curves and leakage currents below $2 \mathrm{nA}$ are the result of our reliable fabrication technique. The hole mobilities and dielectric constants of the ALD oxides are in good agreement with expected values from the literature. We also have proposed a scaling method of the wire conductance using the full top perimeter width in liquid gating experiments, which leads to a constant scaled transconductance for all the wires. The excellent sensing and electrical properties of wires in the $100 \mathrm{~nm}$ range prove the feasibility of a dense integration of SiNW arrays, which is demanded for future systems with multiplexing functionality. For multiplexed detection, we suggest differential measurements with a passivated NW as reference wire, in order to minimize disturbances of the signal, such as drift, temperature or nonspecific adsorption.

\section{Acknowledgement}

This work was supported by the Swiss federal program Nano-Tera, the Swiss National Science Foundation (SNSF), the Swiss Nanoscience Institute (SNI), and the European Commission under the FP7-NMP project Hysens (263091).

\section{References}

1 P. Bergveld: IEEE Trans. Biomed. Eng. BME-17 (1970) 70.

2 P. Bergveld: Sens. Actuators, B 88 (2003) 1.

3 Y. Cui, Q. Q. Wei, H. K. Park and C. M. Lieber: Science 293 (2001) 1289.

4 X. T. Vu, J. F. Eschermann, R. Stockmann, R. GhoshMoulick, A. Offenhäusser and S. Ingebrandt: Phys. Status Solidi A 206 (2009) 426.

5 S. Chen, J. G. Bomer, E. T. Carlen and A. van den Berg: Nano Lett. 11 (2011) 2334.

6 S. Zafar, C. D'Emic, A. Afzali, B. Fletcher, Y. Zhu and T. Ning: Nanotechnology 22 (2011) 405501.

7 Y. Cui, Z. Zhong, D. Wang, W. U. Wang and C. M. Lieber: Nano Lett. 3 (2003) 149.

8 Y. Cui and C. M. Lieber: Science 291 (2001) 851. 
9 G. F. Zheng, F. Patolsky, Y. Cui, W. U. Wang and C. M. Lieber: Nat. Biotechnol. 23 (2005) 1294.

10 B. Tian, T. Cohen-Karni, Q. Qing, X. Duan, P. Xie and C. M. Lieber: Science 329 (2010) 830.

11 E. Stern, J. F. Klemic, D. A. Routenberg, P. N. Wyrembak, D. B. Turner-Evans, A. D. Hamilton, D. A. LaVan, T. M. Fahmy and M. A. Reed: Nature 445 (2007) 519.

12 N. Elfström, R. Juhasz, I. Sychugov, T. Engfeldt, A. E. Karlstrom and J. Linnros: Nano Lett. 7 (2007) 2608.

13 J. H. Ahn, S. J. Choi, J. W. Han, T. J. Park, S. Y. Lee and Y. K. Choi: IEEE Trans. Nanotechnol. 10 (2011) 1405.

14 N. Elfström and J. Linnros: Proc. 17th Int. Vacuum Congress/13th Int. Conf. on Surface Science/Int. Conf. on Nanoscience and Technology, Vol. 100 (J. Phys. Conf. Ser., Bristol, 2008).

15 N. Elfström, A. E. Karlström and J. Linnros: Nano Lett. 8 (2008) 945.

16 X. P. A. Gao, G. Zheng and C. M. Lieber: Nano Lett. 10 (2010) 547.

17 O. Knopfmacher, A. Tarasov, W. Fu, M. Wipf, B. Niesen, M. Calame and C. Schönenberger: Nano Lett. 10 (2010) 2268.

18 O. Knopfmacher, A. Tarasov, M. Wipf, W. Fu, M. Calame and C. Schönenberger: ChemPhysChem 13 (2012) 1157.

19 G. S. May and S. M. Sze: Fundamentals of Semiconductor Fabrication (Wiley, New York, 2007) 1st ed.

20 H. Kim, P. C. McIntyre and K. C. Saraswat: Appl. Phys. Lett. 82 (2003) 106.

21 L. Zhang, H. C. Jiang, C. Liu, J. W. Dong and P. Chow: J. Phys. D: Appl. Phys. 40 (2007) 3707.

22 A. Tarasov, W. Fu, O. Knopfmacher, J. Brunner, M. Calame and C. Schönenberger: Appl. Phys. Lett. 98 (2011) 012114.

23 J. P. Colinge: Silicon-on-Insulator Technology (Springer, Berlin, 2004) 3rd ed.

24 S. M. Koo, Q. L. Li, M. D. Edelstein, C. A. Richter and E. M. Vogel: Nano Lett. 5 (2005) 2519.

25 J. Goldberger, A. I. Hochbaum, R. Fan and P. Yang: Nano Lett. 6 (2006) 973.

26 J. F. Conley, Y. Ono, R. Solanki, G. Stecker and W. Zhuang: Appl. Phys. Lett. 82 (2003) 3508.

27 J. R. Watling, L. F. Yang, A. Asenov, J. R. Barker and S. Roy: IEEE Trans. Device Mater. Reliab. 5 (2005) 103.

28 A. Tarasov, M. Wipf, K. Bedner, J. Kurz, W. Fu, V. A. Guzenko, O. Knopfmacher, R. L. Stoop, M. Calame and C. Schönenberger: Langmuir 28 (2012) 9899. 